\title{
In Response to COVID-19: Promoting Sustainability and Resilience Regarding Major Health Crises
}

\author{
Efstathia Laina* \\ LL.M.; Lawyer, Thessaloniki, Greece; and frequent contributor to EPL
}

The current COVID-19 pandemic is reportedly caused by a zoonosis, a disease naturally transmissible from animals to humans. The pandemic is a vivid example of how human pressure on nature is exposing humans to grave health risks, with mounting social and economic impacts. Experts armed with a solid body of science-based evidence have long alerted that a COVID-19 type of crisis would happen. The remarkable economic growth experienced during the last half century has disrupted ecosystems through widespread land degradation; unplanned urbanisation; expansion of human settlements; rampant deforestation; human-induced climate change; habitat loss; and overexploitation of wildlife. As road-building, logging, mining and other economic activities encroach deeper into nature, people interact more closely with wildlife, and pathogens can more easily cross over from animals to humans.

This article addresses GEF's reported efforts to respond to the zoonotic aspects of COVID-19.

\section{Responses to Zoonotic Diseases}

The only lasting solutions to emerging zoonotic diseases, for which humans have no prior immunity, are to understand their root causes; find workable

${ }^{*}$ Corresponding author. E-mail: stlaina@gmail.com. immediate solutions; work hard to anticipate and prevent future crises; and promote transformational change which can only be achieved through sustainable, inclusive, resilient, low-carbon, low-polluting, nature-positive and circular-economy-based pathways. This logic has been at the core of the GEF's strategy, a logic reinforced and further validated by COVID-19. ${ }^{1}$

The Global Environment Facility (GEF), one of the most significant and reliable funding resources for environmental areas other than climate change, is the principal financial mechanism for the Convention on Biological Diversity and an important financial mechanism for the United Nations Framework Convention on Climate Change, the Stockholm Convention on Persistent Organic Pollutants, the United Nations Convention to Combat Desertification, and the Minamata Convention on Mercury. Working through its 18 agencies, the GEF has provided close to US \$20 billion in grants and mobilised an additional US $\$ 107$ billion in co-financing for more than 4,700 projects in 170 countries. The GEF has also funded projects in international waters and sustainable forest management, supporting implementation of global and regional multilateral environmental agreements. A significant portion of GEF's on-going work accelerates transformational change, for example through the Good Growth Partnership (GGP); and its Impact Programs on Food Systems, 
Land Use and Restoration (FOLUR), Sustainable Cities and Sustainable Forest Management.

The GEF projects and activities have always addressed global environmental challenges, underscoring the health-environment nexus; the importance of science-based policy; the fragility of economic and social systems; and the need to adopt global solutions to address global problems. Thus, there is no immediate need for the GEF to repurpose projects or change project objectives to respond to the COVID-19 crisis; however, there is no guarantee that post-crisis efforts will not threaten the sustainability gains made through current GEF investments. For this reason, the GEF, based on lessons learned from implementation, should take additional actions in the immediate, medium and longer term, with a view to protecting these hard-fought gains, and laying the foundation for a sustainable post-COVID-19 recovery.

\section{Lessons Learned from Evaluation}

The GEF has weathered several health and economic crises during its 27-year history, producing many lessons to inform current efforts. Independent evaluations of GEF programmes can offer valuable insights relevant to the pandemic, based on lessons learned from implementation. In May 2020, the GEF's Independent Evaluation Office (IEO) published a report discussing the GEF's response to the crises, drawing on on-going and completed evaluations, and advising on crisis mitigation strategies and project-building. ${ }^{2}$

An on-going IEO analysis of the GEF projects has found that global crises such as a pandemic are not included in project design, as such events have been extremely rare. Project-design documents focus on risks more related to local systemic issues such as corruption, lack of trust in the government, or lack of interest by financial institutions or other key stakeholders. Not surprisingly, crises are usually mentioned as something to be avoided rather than mitigated. However, the lack of plans for quickly reacting to unforeseen or unpreventable crises could leave projects without adequate guidance when crises - such as the current pandemic - do occur.

\subsection{The GEF's Global Wildlife Program}

The onset of COVID-19 has highlighted the issue of illegal wildlife trade as a pathway for transmitting zoonotic diseases from wildlife to humans. Supply chains and transportation of wildlife and wildlife products from source areas to distant markets, through local, national and international networks, as well as unsafe handling and other practices, allow for natural spill-over and spread. These conditions and practices are even more challenging to monitor - and impossible to regulate - in illegal wildlife trade, which is the fourth most profitable illegal industry in the world, generating US \$26 billion per year.

Several GEF-funded projects include activities related to combating illegal wildlife trade. The Global Partnership on Wildlife Conservation and Crime Prevention for Sustainable Development, launched in 2015, was the first concerted effort to tackle illegal wildlife trade in a coordinated and comprehensive manner. Covering 19 countries in Africa and Asia, the programme aims to prevent the extinction of threatened species by supporting protected area management; reducing poaching of target species; engaging communities in managing human-wildlife conflict; curbing trafficking; reducing demand; improving performance across the enforcement and criminal justice chain; establishing partnerships; and promoting knowledge management.

IEO conducted a formative assessment of the programme in 2017, while the programme was in its early stages of implementation. The lessons learned from evaluation allowed for timely improvements in implementation, underscoring the need for a comprehensive approach along the entire supply chain - from source areas to global markets.

IEO pointed out that greater attention should be paid to a broader spectrum of illegally traded species. A recent study found that the propensity to transmit a zoonotic disease varies minimally among animal groups. Thus, paying attention to more species can make a difference in public health surveillance and zoonotic risk assessment. Since most programmes are found to be limited to certain endangered species and megafauna, the GEF should address this gap by including additional species in its interventions.

Besides, IEO pointed out that most interventions typically focus on reducing poaching in source countries and banning trafficking of illegal wildlife and derived products. Only a few initiatives focus on reducing demand for illegally traded wildlife in Asia, the EU and the US. Thus, interventions should focus more on demand reduction, including public 
awareness and behaviour-change campaigns, because as long as there is demand for wildlife products, there will be trade. At the same time, since poverty and absence of sustainable livelihoods drive communities to engage more in poaching, efforts should also focus on the livelihood security of people living near wildlife.

Furthermore, IEO underscored that combatting illegal wildlife trade, as an international issue, requires a more cohesive approach, including regional initiatives and cross-border activities; and strong political will to address corruption and help strengthen legislation and law enforcement.

\subsection{Health and Socio-economic Outcomes}

GEF interventions in all focal areas biodiversity, sustainable forest management, international waters, climate change, chemicals and waste, and land degradation - help improve human health with mounting social and economic impact. However, since GEF projects are designed to increase global environmental benefits, they usually do not measure human or health co-benefits, though such benefits are often noted as unintended positive outcomes. More recently, the IEO has begun to deliberately measure the socio-economic outcomes of GEF interventions to demonstrate the strong health-environment nexus.

As a result of globalisation and the spread of chemical manufacturing industries, the burden of disease due to pollution is significant and increasing in lower and middle-income countries. The primary objective of the typical GEF chemicals and waste intervention is the elimination of harmful chemicals from the environment, directly affecting human health and wellbeing, and decreasing the disease burden and mortality. This is not a trivial finding, as the conservative global estimate of observable pollution-related deaths in 2015 is 9 million - or 16 percent of total deaths. However, there is always room for improvement.

In artisanal gold mining, for example, GEF interventions included hands-on training, providing healthcare workers with the capacity to assess cases of mercury poisoning in a timely fashion and effectively manage them. Protocols were established to send any unclear diagnoses to the hospital. Success in building proper community health surveillance supplemented efforts to raise awareness of the consequences of mercury use and exposure to health, mobilising the community. Personal health impacts proved to be a great motivating factor to individuals on the ground. Awareness-raising, education and the dissemination of knowledge reduced exposure to risk and inequities in health. At the same time, local stakeholder engagement demonstrated that formalisation of the artisanal and small-scale gold mining (ASGM) industry was a critical step for reducing risk and achieving long-term sustainability.

\subsection{Case Studies and Lessons Learned}

There are examples of GEF projects that have demonstrated resilience through disaster risk management. The IEO report presents some of these examples, aiming at drawing generic lessons that can be applied to new contexts created by crises.

\subsubsection{Crisis Management in the Artisanal Gold Mining Sector}

The GEF's planetGOLD Programme is an eight-country, eight-project initiative aimed at reducing emissions of mercury from ASGM. The programme has been cataloguing the effects of the pandemic on the countries and regions in which it works via its website. The impacts have varied by country, but patterns have emerged. Since mining areas are generally rural, they have not (as of mid-April 2020) been greatly affected by the virus. However, supply chains have been halted through curbs on transportation, and miners have been forced to stop their activities because of government-mandated curfews and closures of non-essential businesses (which in many countries include ASGM). In addition, gold buyers are offering lower prices to miners than before the pandemic even though the international price of gold has not dropped significantly. Consequently, miners, many of whom live in poverty, have not been able to produce as much gold and have to sell what they produce at cheaper prices, earning significantly less income in this time of crisis.

The National Program for the Environmental Sound Management and Live Cycle Management of Chemical Substances project in Ecuador, accelerating non-ASGM activities directly related to healthcare, has collected donations from project 
staff to purchase baskets of basic goods that have been delivered to over 100 families in one of their ASGM project sites, targeting members of a women's group that sorts mine tailings for residual gold. Other projects have released educational videos on public health guidance and outlined ways in which governments could assist artisanal miners to lessen the economic impact of the pandemic.

The IEO report highlighted the proactive programme management and the flexibility of the programme staff that had rapidly adjusted its activities to help affected ASGM communities, maintaining regular updates on the consequences of the pandemic, and soliciting recommendations for ways to respond.

\subsubsection{Crisis Management in the Small and Medium-sized Enterprise Sector in India}

The Promoting Energy Efficiency and Renewable Energy in Selected Micro, Small and Medium Enterprises (MSME) Clusters project in India aims to develop and promote a market environment for energy-efficient and renewable energy technologies in energy-intensive MSME clusters, such as the dairy, ceramics and foundry industries.

The project faced a shock during the COVID-19 pandemic, as MSME resources were fast being depleted in terms of working capital, skilled workforce, inventories and orders. The United Nations Industrial Development Organization (UNIDO) reached out to 85 MSMEs to take stock of their perceived challenges, expectations and plans for recovery. UNIDO contributed to on-going infection prevention and control, while working with the MSMEs to help them get back into business at the earliest possible point in a strategic and planned manner. Tools that have been developed include training programmes, resource materials, and the dissemination of good practices on successful energy-efficient technologies that can reduce energy consumption and increase profitability. Some energy service company models for various potential technologies are also being explored so as to make implementation easier and more attractive to MSMEs in the current crisis.

The report highlighted the importance of staying in touch with project beneficiaries; making an effort to understand their constraints; being able to adjust the assistance provided, as well as technologies and interventions, in a timely fashion; and developing training materials and awareness programmes on disaster management and beneficiaries' preparedness.

\subsubsection{Building Fisher Communities' Resilience During the Ebola Crisis}

The Ebola epidemic, which began in Guinea in late 2013 and spread to Sierra Leone and Liberia, affected the means of making a living for millions of the poorest and most vulnerable people in the region. The areas hit particularly hard by the epidemic were among the most agriculturally productive regions of the three affected countries. Mandatory quarantine measures and fear of infection kept farmers from attending to their fields, resulting in considerable disruption of farming activities. Food trade between villages and bordering countries was slowed, which translated into food shortages. When agricultural lands were abandoned, the fishing industry helped feed the population.

Liberia and Sierra Leone were part of the initial phase of the GEF-funded West Africa Regional Fisheries Program, implemented by the World Bank from 2010 to 2016, aiming to strengthen the capacity of recipient countries to govern and manage targeted fisheries; reduce illegal fishing; and increase local value-added to fish products.

The project's investments in combatting illegal fishing have had transformative results in Liberia and Sierra Leone. The exclusion of illegal trawlers from the six-mile inshore exclusion zone has opened space for artisanal fishers and thus, coastal communities experienced a change in fish availability, increasing their incomes. Fish as a food source started playing a more central role in securing a protein supply for both coastal and inland communities, mostly in the form of smoked small pelagic fish transported by road through a marketing chain controlled by women fish smokers on the coast.

GEF recognised that marine fish resources represented valuable natural capital that could enhance communities' resilience in times of crisis. To help ensure the livelihoods and food security of fisher communities, the GEF approved an additional grant of US \$10 million in 2016 to Guinea, Liberia and Sierra Leone for further support of their fisheries sector, as all three countries had prioritised conservation of their fish stocks for artisanal fisheries in their Ebola recovery plans. 
This case study, based on the World Bank's 2017 implementation completion report, ${ }^{3}$ illustrates the GEF's continuous support in building resilience, and responding to country needs for immediate recovery and long-term food security. Flexibility and quick adaptation, along with long-term strengthening of governance, ensured long-term sustainability in the region.

\subsubsection{Dealing with Infectious Waste during the Ebola Crisis}

During the 2014-16 Ebola outbreak, Guinea, Liberia and Sierra Leone faced challenges in safely disposing of a growing amount of infectious waste. The waste generation rate was estimated at 240 litres of infectious waste per Ebola patient per day. The infected medical equipment and waste had to be properly treated to minimise the risk of transmission. However, in many hospitals and community care centres, Ebola-contaminated waste was burned in barrels, burial pits, or low-tech incinerators that emitted dangerous fumes and created toxic ash.

With support from the GEF and the UN Development Programme (UNDP), environmentally friendly sterilising equipment was used to help dispose of the vast amounts of infectious waste. This equipment, the autoclave, was the first of its kind used in any of the Ebola-affected countries. Unlike burning or incinerating, the autoclave uses high temperature and pressure steam to disinfect the waste, allowing for safe disposal. It does not generate pollutants and has a much smaller carbon footprint. The autoclave was designed under a GEF-funded project, Demonstrating and Promoting Best Techniques and Practices for Reducing Health-Care Waste to Avoid Environmental Releases of Dioxins and Mercury, implemented by UNDP in partnership with the World Health Organization and the non-governmental organisation Health Care Without Harm from 2007 to 2012, in Argentina, India, Latvia, Lebanon, the Philippines, Senegal, Tanzania and Viet Nam.

Since the microbiological tests indicated that the virus was effectively destroyed by the autoclave system, another project was immediately launched by UNDP, providing 20 autoclaves to the three Ebola-affected countries. These autoclaves are now used for treating hospital waste in the post-Ebola recovery period. As a result of this project, non-incineration healthcare waste treatment technologies and mercury-free medical devices were introduced in four Sub-Saharan African countries - Ghana, Madagascar, Tanzania and Zambia - to reduce harmful releases from the health sector.

The report highlighted the synergy between healthcare and environmental protection, leading to overall reduction in harm to both human health and the environment. This GEF project adapted existing technologies to a new crisis situation, leading to a significant and positive change in the Ebola-affected countries, long sustained after the crisis has ended. The initial capital investment and start-up costs for the use and application of new technologies could not be covered by national budget allocations, due to severe budget constraints at the national level. Funding from the GEF, in addition to support provided by project co-financers, was critical for putting in place environmentally sound practices for healthcare waste management and treatment.

\section{Actions to Be Taken to Respond to Crises such as COVID-19}

As a partnership, the GEF has responded to the current crisis by appropriately mapping the potential impacts of the pandemic on GEF-financed projects particularly those in execution - including the impact on project beneficiaries. It has also adjusted timelines and promoted flexibility.

Responding to a brief survey, GEF agencies pointed out that as the situation is evolving, continuous information is needed; and encouraged the GEF Secretariat to coordinate with its partner agencies to mitigate risk to the portfolio; redirect resources in response to the crisis; share examples and best practices of incorporating potential major health crises (including pandemics) or economic/financial crises into project risk matrixes; contribute to understanding the roots of the pandemic; and demonstrate how promoting global environmental benefits can enhance our collective capacity to avoid and manage future crises.

Working to adjust projects and activities to the COVID-19 context, the GEF Secretariat prepared a paper which was presented to the 58th GEF Council Meeting (held virtually from 2-3 June 2020), identifying a set of immediate, medium and longer-term actions to anticipate and prevent future crises. $^{4}$ 


\subsection{Immediate Actions}

\subsubsection{Effectively Tackling Wildlife Trafficking and Consumption}

The GEF Secretariat suggested that through the Global Wildlife Program (GWP), the largest global effort to date to tackle wildlife trafficking, the GEF provide additional financial support to key developing countries in Africa and Asia to help them invest in innovative solutions and effectively address illegal wildlife trade. GWP should also focus on efforts to reduce demand and unchecked consumption of bushmeat and wildlife products; and promote public awareness and behavioural change. The ban on the consumption and farming of wild animals across China offers a unique opportunity to call the attention of citizens worldwide to the implications of buying wild animals for food and medicine; and to put an end to the uncontrolled selling and consumption of wild animals in markets across Africa and Asia.

Furthermore, the GWP could be used as a platform to gather and disseminate information on the ecological and economic consequences of the pandemic; and identify effective ways to address it.

\subsubsection{Conducting Expert Analyses}

To support GEF's response to COVID-19, the GEF Secretariat is seeking advice from a group of experts from like-minded institutions to help the GEF adjust to the crisis context; fill gaps in dealing with wildlife trade and consumption; further support effective protected area management; explore how relevant projects could be fitted with campaigns on public awareness and behaviour change; and gather and disseminate information on the ecological and local economic consequences of pandemics. The expert group will support the development of a white paper, assessing the future risks linked to emerging zoonotic diseases; identifying their root causes; and exploring their connection with deforestation and ecosystem fragmentation.

\subsubsection{Identifying Risks Seriously Compromising Past Gains and Future Outcomes}

Delay in project implementation due to the pandemic is already being reported by agencies. Some 60 percent of the projects in Africa will require short-term adjustments to avoid reversal of achievements, while GWP projects in Asia will require short-term interventions to ramp up work on demand reduction, behavioural change campaigns and high-risk wildlife markets management.

The suspension or inability to perform certain GEF-funded project and programme activities may seriously affect the existing gains and outcomes of these projects or impede the successful achievement of global environmental benefits. Gains achieved under these projects could be lost if measures are not taken quickly and decisively. The GEF Secretariat should continue engaging with implementing agencies to explore how important operations, such as those performed by rangers and other essential personnel in the target protected areas, could be sustained during this difficult period. This will help to avoid creating a vacuum in law enforcement that could be used by poachers, as has already been reported with rhinos in South Africa.

\subsection{Medium-term Actions}

In order to ensure that current and future projects and programmes under GEF-7 can integrate the risks and opportunities that have arisen in the months since COVID-19 appeared, the GEF Secretariat will work on a blueprint to guide GEF's actions for the next two years, or the remainder of the GEF-7 cycle, benefitting from the upstream dialogue and initial assessment being conducted with GEF agencies.

The blueprint will address key aspects of on-going work and explore how the crisis is affecting strategic platforms of engagement on themes like food security (GGP, FOLUR), cities (Sustainable Cities), mining and mercury use (planetGOLD), and circular economy projects. Important underlying factors brought about by COVID-19 will also be analysed, ranging from changes in fossil-fuel prices and impacts on emissions, likelihood of fossil-fuel-based (re)development and infrastructure needs, impacts on plastics and waste management, food (in)security, changes in consumption patterns, and impacts on and from global commodities supply chains. Thus, the blueprint will lay the policy groundwork for the development and application of green recovery tools and approaches.

As the GEF Secretariat starts considering the new strategy that will be presented to the GEF Participants Group to orient the GEF-8 cycle of 
investments, the blueprint can help with providing preliminary information and early exploratory guidance on what types of project design features and principles can enhance the likelihood of a sustainable post-crisis recovery pathway.

As any recovery effort must be built with resilience at its core, the blueprint will encompass the GEF's highly relevant programme on adaptation funded by the Least Developed Countries Fund and the Special Climate Change Fund. Besides, the successful Challenge Program for Adaptation Innovation funded under the GEF-7 Adaptation Strategy will be further harnessed to more rapidly identify new concepts directed at promoting adaptability and building up the resilience of communities and businesses in the time of crises.

The blueprint can also delve into other areas of relevance for the crisis, such as through the Chemicals and Waste focal management portfolio. During the COVID-19 pandemic, infectious healthcare waste is a rapidly increasing waste stream. Developing countries find themselves in the dire situation of mounting infectious healthcare waste, overwhelming existing waste management capacity. This situation calls for the GEF agencies to intervene. Otherwise, the unintentional release of persistent organic pollutants, mercury and other hazardous chemicals will experience surges when wastes which are not sorted/collected properly, are burned in the open air or in low-tech incinerators. Interventions can also play an important role in protecting waste workers and healthcare staff who may be at risk of (re)infection due to unsound handling of healthcare waste.

Hopefully, the analysis will also include specific guidance tailored to decision-makers who have a strong influence on the possible recovery pathways that will follow the crisis.

\subsection{Long-term Actions}

In less than one year, the GEF will start to develop new strategies to guide the Eighth Replenishment cycle. At its core, COVID-19 has resulted from the collision between human systems and natural systems; and the fundamental solution to this problem is to transform human systems and re-align them with more sustainable practices. The GEF has been promoting system change through GEF-6 and GEF-7, but more is needed. As the strategic discussions for GEF-8 begin, information about the origins and consequences of COVID-19, as well as crisis mitigation tools and strategies, should be collected and disseminated. Furthermore, the GEF should continue engaging with multi-stakeholder platforms to promote transformation. Such an engagement could provide guidance on how domestic and international recovery programmes can be steered towards nature-based, low-carbon, resilient and safer infrastructure and systems.

\section{Conclusions}

The current pandemic has highlighted the need to accelerate progress toward sustainability, developing responsive business and economic models, and identifying opportunities for "building back better". Climate-change scenarios predict that we will see an increase in global, highly interruptive shocks over the next 10-20 years. If we do not learn from this pandemic, if we do not change our behaviour today, similar multidimensional crises with environmental, health and socio-economic impacts are likely to recur. The GEF projects and activities aim at promoting environmental sustainability and resilience, aiming at mitigating the current crisis consequences; and preventing and effectively managing future crises.

\section{Endnotes}

1 The GEF's COVID-19 Response Page is available at https://www.thegef.org/news/gefs-response-covid-19. COVID-19 updates from the GEF partnership are available at https://www. thegef.org/news/covid-19-updates-gef-partnership.

${ }^{2}$ The IEO's report, entitled "The GEF response to the Crisis What can we learn from Evaluation?", is available at https://www.gefieo.org/documents/gef-response-crisis-what-canwe-learn-evaluation. "Lessons for COVID-19 from GEF IEO Evaluations" is available at https://www.gefieo.org/news/lessonscovid-19-gef-ieo-evaluations.

3 World Bank. 2017. "Implementation and Completion Results Report". Available at https://documents.worldbank.org/ en/publication/documents-reports/documentdetail/671791496156 189517/africa-first-phase-of-west-africa-regional-fisheries-progr am-project.

${ }^{4}$ The information document prepared by the GEF Secretariat for the $58^{\text {th }}$ GEF Council Meeting, outlining its planned response to COVID-19, is available at https://www.thegef.org/sites/default/ files/council-meeting-documents/EN_GEF_C.58_Inf.07_GEF\%27 s\%20Response \%20to\%20COVID-19.pdf. 